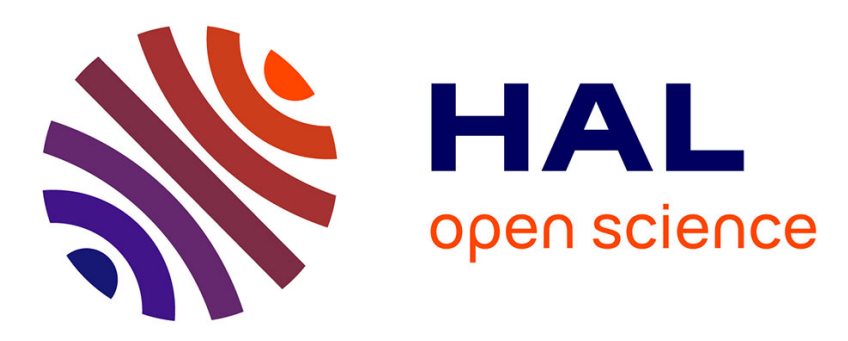

\title{
Bifurcation and creep effects in a viscoelastic non-local damageable continuum
}

\author{
Theocharis Baxevanis, Gilles Pijaudier-Cabot, Frédéric Dufour
}

\section{To cite this version:}

Theocharis Baxevanis, Gilles Pijaudier-Cabot, Frédéric Dufour. Bifurcation and creep effects in a viscoelastic non-local damageable continuum. European Journal of Mechanics - A/Solids, 2008, 27, pp.548-563. 10.1016/j.euromechsol.2007.11.009 . hal-00333866

\section{HAL Id: hal-00333866 https://hal.science/hal-00333866}

Submitted on 24 Oct 2008

HAL is a multi-disciplinary open access archive for the deposit and dissemination of scientific research documents, whether they are published or not. The documents may come from teaching and research institutions in France or abroad, or from public or private research centers.
L'archive ouverte pluridisciplinaire HAL, est destinée au dépôt et à la diffusion de documents scientifiques de niveau recherche, publiés ou non, émanant des établissements d'enseignement et de recherche français ou étrangers, des laboratoires publics ou privés. 


\title{
Bifurcation and creep effects in a viscoelastic
}

\section{non-local damageable continuum}

\author{
Theocharis Baxevanis ${ }^{1}$ \\ Department of Applied Mathematics University of Crete, Heraklion 71409 \\ Heraklion, Greece
}

\begin{abstract}
Gilles Pijaudier-Cabot
ERT R\&DO, Institut de Recherche en Génie Civil et Mécanique, Centrale Nantes, CNRS, Université de Nantes, 1 rue de la noё, BP 92101, 44321 Nantes cedex 3, France
\end{abstract}

Frédéric Dufour

ERT REDO, Institut de Recherche en Génie Civil et Mécanique, Centrale Nantes, CNRS, Université de Nantes, 1 rue de la noë, BP 92101, 44321 Nantes cedex 3, France

\begin{abstract}
The conditions for localization in a material described by a non-local damage-based constitutive relation coupled with a Kelvin type creep relation are derived in a closed form. The inception of a localized mode is considered as a bifurcation into
\end{abstract}


a harmonic mode. The criterion of bifurcation is reduced to the classical form of singularity of a pseudo acoustic tensor; this tensor involves the ratio between the elasto-damage strain and the total one at the inception of localization and the wavelength of the bifurcation mode through the Fourier transform of the weight function used in the definition of non-local damage. A geometrical approach was adopted to analyze localization. The proposed coupled model preserves the properties of localization limiters; the minimum wavelength of the localization modes cannot be zero. This wavelength increases when the material parameter $\alpha(0 \leq \alpha \leq 1)$, which is the fraction of creep strain entering into the evolution of damage, is decreasing. Moreover, the normal $\boldsymbol{n}$ to the localization band depends only upon the elastodamage state of strain. Under some conditions on the growth of the yield function of damage, the wavelength decreases as the creep strain increases. The proposed model fails to predict creep rate-effects when damage is supposed, as it is common practice, to be driven solely from the elastic release energy $(\alpha=0)$.

Key words: Bifurcation, non-local constitutive laws, size effects, creep, damage

Email addresses: theocharis@tem.uoc.gr (Theocharis Baxevanis), Gilles.Pijaudier-Cabot@ec-nantes.fr (Gilles Pijaudier-Cabot), Frederic.Dufour@ec-nantes.fr (Frédéric Dufour).

1 This work was partially supported from the EU project 'Degradation and Instabilities in Geomaterials with Application to Hazard Mitigation' (DIGA-HPRN-CT2002-00220) in the framework of the Human Potential Program, Research Training Networks. 


\section{Introduction}

The growth of micro-cracks in progressively fracturing rate-independent materials is a fairly distributed process which is associated with stable material response. For certain stress trajectories, however, a different deformation mode may prevail, consisting of formation of discrete failure planes (macro-cracks). In the latter case, the mechanical response as observed at the macroscale becomes, in general, unstable. The inception of a localized mode may be considered as a bifurcation problem related to the loss of positive definiteness of the tangent material stiffness operator governing the homogeneous deformation (Rudnicki and Rice, 1975; Rice, 1976). In mathematical terms, the elliptic character of the set of partial differential equations governing equilibrium is lost (ill-posedness of the related boundary value problem). This result was derived for the linearized rate equation problem, considering what is commonly denoted as a linear comparison solid (Hill, 1959). Loss of ellipticity may result in a discontinuous rate of deformation and constant total energy consumption. Path stability considerations indicate that this particular solution is expected and consequently failure occurs without energy dissipation (Bažant, 1988). In dynamics, the situation is similar. The hyperbolic differential equations of motion may become parabolic or elliptic and consequently the initial-boundary value problem ill-posed (Hadamart, 1903).

Non-conventional constitutive relations, called localization limiters, based on 
sophisticated techniques that enrich the standard continuum, have been adopted by many authors to eliminate the deficiencies caused by ill-posedness of boundary value problems. A wide class of localization limiters is based on the assumption that the stress of a material point is not only determined by the history of strain at this point but also by interactions with other material points (non-local integral continuum). The distance over which interactions are important is related to an internal length that prevents energy dissipation to become zero as failure occurs (Simo and Fox, 1989; Bažant and PijaudierCabot, 1988). Another class of localization limiters relies on enrichment by terms that contain gradients of the state variables. Some of these models deal with strain gradients, i.e. higher-order gradients of the displacement field. In the above theories the rate-independent nature of the material model is preserved. Incorporation of rate-dependent viscous terms can limit localization due to softening (Needleman, 1988; Loret and Prevost, 1990; Dubé et al., 1996). The size of the localization zone, however, is controlled by this rate effect and it may not be possible to fit, with the same expression of the evolution of damage or viscoplastic strains, the rate effect on the stress-strain response and the width of the localization zone that is consistent with experimental observations.

In this contribution, attention is focused on concrete structures and we are interested in predicting the failure due to localization of damage of those structures. In common practice, it is usually assumed for concrete that lin- 
ear viscoelasticity takes place for low stress levels where the instantaneous mechanical behavior is elastic. In the contrary, for high stress levels the linearity hypothesis fails. Bazant and co-workers (Bažant, 1993) have shown that creep at high stress levels cannot be captured merely by a non-linear generalization of viscoelastic stress-strain relation. Experimental evidence associate creep strains at high stress levels to microcracking nucleation and growth, i.e. to damage (Rüsch was the first one to conduct such experiments (Rüsch, 1960)). As recalled by Bazant and Planas (Bažant and Planas, 1998), timedependent fracture of concrete is caused by viscoelasticity of the material, and (or) breakage of bonds in the fracture process zone. The difference between these two effects can be observed on structural responses and size effect. In the range of quasi-static loading, that is, in the absence of inertia forces and wave propagation effects, viscoelasticity in the bulk (linear creep) causes an increase of the bearing capacity for increasing loading rates, with a decreasing peak displacement. For geometrically similar structures of different sizes and mode I crack propagation, the set of data points corresponding to the nominal stresses at failure shifts toward increasing brittleness on the size effect plot (Bažant and Planas, 1998), upon a decrease of the loading rate. It means that the set of data points are getting closer to the LEFM criterion (Figure (B.1)). The size of the fracture process zone decreases (see e.g. (Haidar et al., 2005)). This shift has been observed experimentally by Bazant and Gettu (Bažant and Gettu, 1992; Bažant and Li, 1997), as shown in Figure (B.1). On the other hand, if the rate dependence is caused only by bond breakages, the peak loads 
correspond to increasing displacements and there is no shift of this kind on the size effect plot. Note that ductility and brittleness are defined here, and in the remaining, as the position of the experimental data with respect to the two asymptotic criteria: strength of materials and LEFM (see Figure (B.1)). Ductility increases when the set of data points shift to the left and brittleness increases when they shift to the right.

[Fig. 1 about here.]

Many writers have used coupled models to capture mechanical, viscoelastic and non-linear instantaneous behaviors. In these models, linear viscoelasticity is coupled to a rate independent elasto-plastic model (de Borst et al., 1993), to a smeared crack model (Rots, 1988) or to a damage model (Mazzotti and Savoia, 2003). The aim of this paper is to investigate the condition of localization for such a coupled model and to see the influence of creep on failure. Since the influence of viscoelasticity on the strength of concrete structures may be of importance (decrease of bearing capacity, increase of brittleness) it is important for engineering practice to check that available constitutive models are capable of predicting such effects.

In the present study we will consider as an example a viscoelastic model coupled to rate independent damage inspired from Omar et al. (Omar et al., 2004), in which the relationship between the effective stress, defined in a standard way according to continuum damage (Lemaitre and Chaboche, 1985), 
and the strain follows a Kelvin chain. The analysis follows the method used by Benallal (Benallal, 1992) for thermo-mechanical problems and PijaudierCabot and Benallal (Pijaudier-Cabot and Benallal, 1993) for the case of a rate independent non-local model. This paper is organized as follows: In Section 2, the creep and damage models are briefly recalled and the rate formulation for creep-damage model is derived based on the small strain assumption. In Section 3, the strain localization analysis is performed. The rate constitutive relations for the linear comparison solid, as well as the bifurcation conditions under the assumption that the solid considered is either infinite or at least large enough so that boundary layers effects can be neglected, are derived. Moreover, the dependence of the admissible wavelength on the parameters of constitutive law and on the state variables is discussed. An illustration is presented in the case of a uniaxial response in Section 4.

\section{Constitutive relation}

\subsection{Creep model}

Consider first a quasi-brittle material, such as concrete, exhibiting non-ageing linear viscoelasticity and characterized by the uniaxial compliance function $J\left(t, t^{\prime}\right)$, representing the uniaxial strain at age $t$ caused by a unit stress enforced

at any age $t^{\prime}$ (the linearity hypothesis agrees very well with test results in which no strain reversals have taken place, in the case of basic creep, and for stress 
levels less than $40 \%$ of the strength limit). The assumption of a non-ageing material will be used throughout this paper for the sake of simplicity, and causes $J\left(t, t^{\prime}\right)$ to be a function of just the time lag $\left(t-t^{\prime}\right)$.

By approximating the compliance function by a Dirichlet series

$$
J\left(t, t^{\prime}\right) \simeq \frac{1}{E}+\sum_{i=1}^{N} \frac{1}{E_{i}}\left[1-\exp \left(-\frac{t-t^{\prime}}{\tau_{i}}\right)\right]
$$

where $\tau_{i}, i=1,2, \ldots . N$, are fixed parameters called retardation times, $E$ is Young's modulus and $E_{i}, i=1,2, \ldots N$, are age-independent moduli which can be determined by least-square fitting to the "exact" compliance function, it can be proved that

$$
\epsilon^{t o t}(t)=\epsilon^{e l, d}(t)+\sum_{i=1}^{N} \epsilon_{i}^{c r}(t), \quad i=1,2, \ldots N
$$

where the strains $\epsilon^{e l, d}$ and $\epsilon_{i}^{c r}, i=1,2, \ldots N$, are governed by the following equations

$$
E \epsilon^{e l, d}(t)=\sigma(t)
$$

and

$$
E_{i} \epsilon_{i}^{c r}(t)+\tau_{i} E_{i} \dot{\epsilon}_{i}^{c r}(t)=\sigma(t), \quad i=1,2, \ldots N
$$

The total stress $\sigma$ in (4) is expressed as the sum of two terms. The first term, $E_{i} \epsilon_{i}^{c r}$, corresponds to the stress of an elastic spring of stiffness $E_{i}$. The second term, $\tau_{i} E_{i} \dot{\epsilon}_{i}^{c r}$, is the stress generated by the strain rate $\dot{\epsilon}_{i}^{c r}$ in a linear dashpot of viscosity $\eta_{i}=\tau_{i} E_{i}$. This means that in the non-ageing case the approximation of the compliance function by the Dirichlet series corresponds to a Kelvin 
chain with constant properties of individual elements. The initial conditions for $\epsilon_{i}^{c r}$ are $\epsilon_{i}^{c r}(0)=0$.

Using (2), (3) and (4), we finally obtain the rate form

$$
\dot{\sigma}=E \dot{\epsilon}^{t o t}-E \sum_{i=1}^{N}\left(\frac{\sigma}{\tau_{i} E_{i}}-\frac{\epsilon_{i}^{c r}}{\tau_{i}}\right) .
$$

A generalization of this uniaxial stress-strain constitutive relation is performed under the simplifying assumption that the Poisson ratio for creep is approximately time-independent and about the same as the elastic Poisson ratio $\nu$. Thus, in view of the assumed isotropy and based on the principle of superposition, the following relation holds:

$$
\varepsilon^{t o t}(t)=\mathbf{C}_{\nu}^{-1} J\left(t, t^{\prime}\right) \boldsymbol{\sigma}\left(t^{\prime}\right)+\mathbf{C}_{\nu}^{-1} \int_{t^{\prime}}^{t} J(t, \tau) d \boldsymbol{\sigma}(\tau)
$$

where $\boldsymbol{\sigma}(t)$ is the column matrix of stress at time $t, \boldsymbol{\varepsilon}^{\text {tot }}(t)$ is the column matrix of engineering strain components, and

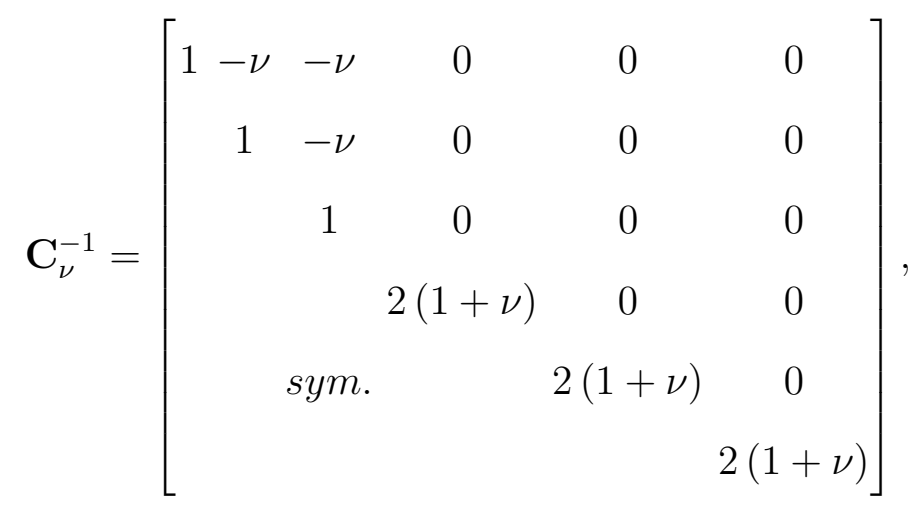

is the unit elastic compliance matrix, corresponding to Young's modulus $E=1$ (for a review see (Bažant, 1993)) and leads to the following incremental form

$$
\dot{\boldsymbol{\sigma}}=E \mathbf{C}_{\nu} \dot{\varepsilon}^{t o t}+E \mathbf{C}_{\nu} \sum_{i=1}^{N} \frac{\varepsilon_{i}^{c r}}{\tau_{i}}-E \sum_{i=1}^{N} \frac{\boldsymbol{\sigma}}{\tau_{i} E_{i}}
$$




\subsection{Non-local damage model}

We will use in the following the scalar continuum damage model proposed by Pijaudier-Cabot and Bazant (Pijaudier-Cabot and Bažant, 1987). The description of the model is that of Pijaudier-Cabot and Benallal (Pijaudier-Cabot and Benallal, 1993). Despite its simplicity (namely the fact that the model response is the same in tension and compression), this model bears the essential characteristics pertaining to a non-local integral model. The constitutive relation reads:

$$
\boldsymbol{\sigma}=(1-D) E \mathbf{C}_{\nu} \varepsilon^{e l, d}
$$

where $D$ is the damage variable and $\varepsilon^{e l, d}$ is the (elastic) strain.

The growth of damage is defined by a loading function $f$

$$
f(\bar{y}, D)=\int_{0}^{\bar{y}} F(z) d z-D
$$

where $F$ is a function deduced from experimental data and $\bar{y}(\boldsymbol{x})$ is the average energy release rate due to damage at point $\boldsymbol{x}$ of the solid

$$
\bar{y}(\boldsymbol{x})=\int_{V} \psi(\boldsymbol{x}-\boldsymbol{s}) y(\boldsymbol{s}) d \boldsymbol{s} .
$$

$V$ is the volume of the structure, $\psi(\boldsymbol{x}-\boldsymbol{s})$ is a normalized weighting function and $y(s)$ is the energy release rate due to damage at point $s$ defined by

$$
y(\boldsymbol{s})=\frac{1}{2} \varepsilon^{e l, d}(\boldsymbol{s}) E \mathbf{C}_{\nu} \boldsymbol{\varepsilon}^{e l, d}(\boldsymbol{s})
$$

The evolution law is prescribed according to the standard format of non- 
associated irreversible processes:

$$
\dot{D}=\phi \frac{\partial g}{\partial \bar{y}}
$$

with the classical Kuhn-Tucker conditions $\phi \geq 0, f \leq 0$ and $\phi f=0$. The dot over a variable indicates differentiation with respect to time, $g$ is the evolution potential controlling the growth of damage and $\phi$ is the damage multiplier. In this paper for the sake of simplicity, we assume $g=\bar{y}$.

The rate form of (8) reads

$$
\dot{\boldsymbol{\sigma}}=(1-D) E \mathbf{C}_{\nu}\left(\dot{\varepsilon}^{e l, d}-\frac{\dot{D}}{1-D} \varepsilon^{e l, d}\right)
$$

\subsection{Rate-type creep-damage law}

The proposed rate-type formulation of a viscoelastic damageable non-ageing continuum assumes a deteriorating Young's modulus E. In fact, the effective stress $\boldsymbol{\sigma}^{t}$ is defined first according to:

$$
\boldsymbol{\sigma}^{t}=\frac{\boldsymbol{\sigma}}{1-D}
$$

Then, it is assumed that the relationship between the effective stress and the total strain follows a linear viscoelastic model. This is a rather classical approach, inspired from Lemaitre and Chaboche (Lemaitre and Chaboche, 1985), which turns out to induce a decrease of the Young's modulus in the 
constitutive relations in our case (without affecting the characteristic times in the Kelvin chain). Consequently, we obtain the following stress-strain relation $\dot{\boldsymbol{\sigma}}=(1-D) E \mathbf{C}_{\nu} \dot{\varepsilon}^{t o t}-\dot{D} E \mathbf{C}_{\nu} \varepsilon^{e l, d}+(1-D) E \mathbf{C}_{\nu} \sum_{i=1}^{N} \frac{\varepsilon_{i}^{c r}}{\tau_{i}}-(1-D) E \sum_{i=1}^{N} \frac{\boldsymbol{\sigma}}{\tau_{i} E_{i}}$

The above model in tensorial notation reads

$$
\dot{\sigma}=(1-D) \mathbb{C}: \dot{\varepsilon}^{t o t}-\dot{D} \mathbb{C}: \varepsilon^{e l, d}+(1-D) \mathbb{C}: \sum_{i=1}^{N} \frac{\varepsilon_{i}^{c r}}{\tau_{i}}-(1-D) E \sum_{i=1}^{N} \frac{\sigma}{\tau_{i} E_{i}}
$$

where the colon denotes the contracted tensorial product, $\mathbb{C}$ is the elastic material stiffness tensor, and by $\mathbb{X}$ we denote the tensorial transcription of the engineering matrix $\boldsymbol{X}$.

The present model is also based on the assumption that only a fraction of the energy release rate due to creep contributes to the damage evolution with time. The motivation for introducing this parameter into the constitutive law is that for low stress levels although creep strain can be large (even larger than that corresponding to peak stress for short term loading) there is no significant variation of the elastic modulus; that is no significant damage (Mazzotti and Savoia, 2001). Hence, we assume that the function $y(\boldsymbol{s})$ defined in (11) is now equal to

$$
y(\boldsymbol{s})=\frac{1}{2}\left(\sigma^{t}(\boldsymbol{s}): \varepsilon^{e l, d}(\boldsymbol{s})+\alpha \sigma^{t}(\boldsymbol{s}): \sum_{i=1}^{N} \varepsilon_{i}^{c r}(\boldsymbol{s})\right),
$$

or equivalently:

$$
y(\boldsymbol{s})=\frac{1}{2}\left(\varepsilon^{e l, d}(\boldsymbol{s}): \mathbb{C}: \varepsilon^{e l, d}(\boldsymbol{s})+\alpha \varepsilon^{e l, d}(\boldsymbol{s}): \mathbb{C}: \sum_{i=1}^{N} \varepsilon_{i}^{c r}(\boldsymbol{s})\right),
$$


where the creep strain is

$$
\sum_{i=1}^{N} \varepsilon_{i}^{c r}=\varepsilon^{t o t}-\varepsilon^{e l, d}
$$

and the coefficient $\alpha$ is such that $0 \leq \alpha \leq 1$.

Consider now an initial state of equilibrium at time $t_{0}$ denoted by the state variables $\varepsilon_{0}^{e l, d}$ and $D_{0}$. The rate constitutive relation describing the behavior of the material from this state is

$$
\dot{\sigma}=\left(1-D_{0}\right) \mathbb{C}: \dot{\varepsilon}^{t o t}-\dot{D} \mathbb{C}: \varepsilon_{0}^{e l, d}+\left(1-D_{0}\right) \mathbb{C}: \sum_{i=1}^{N} \frac{\varepsilon_{i_{0}}^{c r}}{\tau_{i}}-\left(1-D_{0}\right) E \sum_{i=1}^{N} \frac{\sigma_{0}}{\tau_{i} E_{i}}
$$

\section{Strain localization analysis}

\subsection{Equations of motion}

The equations of motion are a set of non-linear integro-differential equations since the constitutive relations themselves are non-linear. Upon linearization of the equation of motion about the initial state $\left(\varepsilon_{0}^{t o t}, \varepsilon_{0}^{e l, d}, D_{0}\right)$, the momentum equations become

$$
\operatorname{div} \dot{\sigma}(\boldsymbol{x})=\rho \frac{\partial^{2} \boldsymbol{v}}{\partial t^{2}}
$$

where $\boldsymbol{v}$ is the time derivative of the perturbation applied to the initial state. Equations (21) are still non-linear due to the constitutive relations. Linearization is now performed under the assumption $\dot{f}=0$. This assumption is classical in the analyses of localization. The solid that follows such a constitutive 
relation is called linear comparison solid (Hill, 1959). Under this assumption and using the damage law (12) and equation (18), the equations of motion become

$$
\begin{aligned}
\operatorname{div}\left(\left(1-D_{0}\right) \mathbb{C}:\right. & \dot{\varepsilon}^{t o t}-\mathbb{C}: \varepsilon_{0}^{e l, d} F\left(\bar{y}_{0}\right) \\
& \int_{V} \frac{1}{2} \psi(\boldsymbol{s})\left(2 \varepsilon_{0}^{e l, d}(\boldsymbol{x}+\boldsymbol{s}): \mathbb{C}: \dot{\varepsilon}^{e l, d}(\boldsymbol{x}+\boldsymbol{s})\right. \\
+\alpha\left(\varepsilon_{0}^{e l, d}(\boldsymbol{x}+\boldsymbol{s}):\right. & \left.\left.\mathbb{C}: \sum_{i=0}^{M} \dot{\varepsilon}_{i}^{c r}(\boldsymbol{x}+\boldsymbol{s})+\sum_{i=0}^{M} \varepsilon_{i_{0}}^{c r}(\boldsymbol{x}+\boldsymbol{s}): \mathbb{C}: \dot{\varepsilon}^{e l, d}(\boldsymbol{x}+\boldsymbol{s})\right)\right) d(\boldsymbol{s}) \\
+ & \left.\left(1-D_{0}\right) \mathbb{C}: \sum_{i=1}^{N} \frac{\varepsilon_{i_{0}}^{c r}}{\tau_{i}}-\left(1-D_{0}\right) E \sum_{i=1}^{N} \frac{\sigma_{0}}{\tau_{i} E_{i}}\right)=\rho \frac{\partial^{2} \boldsymbol{v}}{\partial t^{2}} .
\end{aligned}
$$

The initial state of deformation and damage is assumed homogeneous through out the solid of volume $V$. The volume of the solid is assumed to be large enough so that boundary layer effects introduced by spatial averaging can be neglected.

Let us now consider the propagation of a harmonic wave in the direction defined by $\boldsymbol{n}$

$$
\boldsymbol{v}=\boldsymbol{A} e^{-i \xi(\boldsymbol{n} \cdot \boldsymbol{x}-c t)}
$$

where $\xi$ is the wave number, $c$ is the phase velocity, $\boldsymbol{A}$ is the amplitude of the perturbation and $i$ is the imaginary constant such that $i^{2}=-1$. The resulting rate of deformation is

$$
\dot{\varepsilon}^{t o t}=-\frac{1}{2} i \xi(\boldsymbol{A} \otimes \boldsymbol{n}+\boldsymbol{n} \otimes \boldsymbol{A}) e^{-i \xi(\boldsymbol{n} \cdot \boldsymbol{x}-c t)},
$$

where $\otimes$ denotes the tensorial product. 
This harmonic perturbation is admissible if it satisfies the rate equation of equilibrium. Under the assumption that the state variables $\varepsilon_{0}^{e l, d}$ and $D_{0}$ are constant throughout the solid, taking into account (19) and noticing that $\dot{\varepsilon}_{i}^{\text {cr }}$ defined by

$$
\dot{\varepsilon}_{i}^{c r}=\frac{\sigma_{0}}{\tau_{i} E_{i}}-\frac{\varepsilon_{i_{0}}^{c r}}{\tau_{i}}
$$

depends on the initial state only and is homogeneous over the solid, the following relation is obtained:

$$
\begin{array}{r}
\left(\boldsymbol{n} \cdot\left(1-D_{0}\right) \mathbb{C} \cdot \boldsymbol{n}\right. \\
\left.-\bar{\psi}(\xi \boldsymbol{n}) F\left(\bar{y}_{0}\right)\left(\boldsymbol{n} \cdot \frac{1}{2}(2-\alpha) \mathbb{C}: \varepsilon_{0}^{e l, d} \otimes \varepsilon_{0}^{e l, d}: \mathbb{C} \boldsymbol{n}+\boldsymbol{n} \cdot \frac{1}{2} \alpha \mathbb{C}: \varepsilon_{0}^{e l, d} \otimes \varepsilon_{0}^{t o t}: \mathbb{C} \cdot \boldsymbol{n}\right)\right) \cdot \boldsymbol{A} \\
=\rho c^{2} \boldsymbol{A}, \quad(26)
\end{array}
$$

where $\bar{\psi}(\xi \boldsymbol{n})$ is defined by

$$
\bar{\psi}(\xi \boldsymbol{n})=\int_{V} \psi(\boldsymbol{s}) e^{-i \xi \boldsymbol{n} \cdot \boldsymbol{s}} d(\boldsymbol{s})
$$

In order to obtain (26), one must substitute (23), (24) and (25) into (22). Since the solid is assumed large, $\bar{\psi}(\xi \boldsymbol{n})$ reduces to the Fourier transform of the weighting function. By considering the weighting function as isotropic, $\bar{\psi}(\xi \boldsymbol{n})$ becomes independent of the direction $\boldsymbol{n}$, hence $\bar{\psi}(\xi \boldsymbol{n}) \equiv \bar{\psi}(\xi)$. Equation (26) can be written equivalently as an eigenvalue problem

$$
\left[\boldsymbol{n} \cdot \mathbb{H}^{*} \cdot \boldsymbol{n}-\rho c^{2} \mathbb{I}\right] \cdot \boldsymbol{A}=\mathbf{0},
$$


where $\mathbb{I}$ is the second order identity tensor and

$$
\begin{aligned}
\boldsymbol{n} . \mathbb{H}^{*} \cdot \boldsymbol{n}=\boldsymbol{n} \cdot\left(1-D_{0}\right) \mathbb{C} \cdot \boldsymbol{n} & \\
& -\bar{\psi}(\xi \boldsymbol{n}) F\left(\bar{y}_{0}\right)\left(\boldsymbol{n} \cdot \frac{1}{2}(2-\alpha) \mathbb{C}: \varepsilon_{0}^{e l, d} \otimes \varepsilon_{0}^{e l, d}: \mathbb{C} \cdot \boldsymbol{n}\right. \\
& \left.+\boldsymbol{n} \cdot \frac{1}{2} \alpha \mathbb{C}: \varepsilon_{0}^{e l, d} \otimes \varepsilon_{0}^{t o t}: \mathbb{C} \cdot \boldsymbol{n}\right) .
\end{aligned}
$$

Equation (26) admits non-trivial solutions if and only if

$$
\operatorname{det}\left[\boldsymbol{n} \cdot \mathbb{H}^{*} \cdot \boldsymbol{n}-\rho c^{2} \mathbb{I}\right]=0 .
$$

\subsection{Statics - Solutions at the bifurcation point}

Condition

$$
\operatorname{det}\left[\boldsymbol{n} \cdot \mathbb{H}^{*} \cdot \boldsymbol{n}\right]=0,
$$

is similar to the localization condition in a non-local continuum derived by Pijaudier-Cabot and Benallal (Pijaudier-Cabot and Benallal, 1993). Now, we restrict our analysis for the sake of simplicity to the uniaxial case or to loading paths such that

$$
\varepsilon_{0}^{e l, d}=\kappa_{0} \varepsilon_{0}^{t o t}
$$

where $\kappa_{0}$ is a real such that $0 \leq \kappa_{0} \leq 1$ (Appendix A). Using (32), we obtain after some algebra

$$
\frac{1}{2}\left((2-\alpha) \kappa_{0}^{2}+\alpha \kappa_{0}\right) \boldsymbol{n} \cdot \mathbb{C}: \varepsilon_{0}^{t o t} \cdot(\boldsymbol{n} \cdot \mathbb{C} \cdot \boldsymbol{n})^{-1} \cdot \varepsilon_{0}^{t o t}: \mathbb{C} \cdot \boldsymbol{n}=\frac{\left(1-D_{0}\right)}{\bar{\psi}(\xi \boldsymbol{n}) F\left(\bar{y}_{0}\right.}
$$


Let now,

$$
\psi(\boldsymbol{x})=\psi_{0} \exp \left(-\frac{\|\boldsymbol{x}\|^{2}}{2 l_{c}^{2}}\right)
$$

where $l_{c}$ is the internal length of the non-local continuum and $\psi_{0}$ is a normalizing factor. The Fourier transform of the above is

$$
\bar{\psi}(\xi \boldsymbol{n})=\exp \left(-\frac{\xi^{2} l_{c}^{2}}{2}\right)
$$

Solving (31) consists in finding the normal $\boldsymbol{n}$ and the wavelength $2 \pi / \xi$ satisfying this equation. A geometrical approach proposed by Benallal (Benallal, 1992) for the analysis of localization phenomena in thermo-elasto-plasticity is used here. Under the assumption of constant Poisson ratio

$$
\mathbb{C} \equiv \lambda \delta_{k l} \delta_{m n}+\mu\left(\delta_{k m} \delta_{l n}+\delta_{k n} \delta_{l m}\right)
$$

where

$$
\lambda=\frac{\nu E}{(1+\nu)(1-2 \nu)}, \quad \mu=\frac{E}{2(1+\nu)},
$$

are the Lamé constants. One then gets

$$
\begin{gathered}
(\boldsymbol{n} \cdot \mathbb{C} \cdot \boldsymbol{n})^{-1}=\frac{\mathbb{I}}{\mu}-\frac{\lambda+\mu}{\mu(\lambda+2 \mu)} \boldsymbol{n} \otimes \boldsymbol{n}, \\
\mathbb{C}: \varepsilon_{0}^{t o t} \cdot \boldsymbol{n}=\lambda \operatorname{tr}\left(\varepsilon_{0}^{t o t}\right) \boldsymbol{n}+2 \mu \varepsilon_{0}^{t o t} \cdot \boldsymbol{n}, \\
\boldsymbol{n} \cdot \mathbb{C}: \varepsilon_{0}^{t o t} \cdot \boldsymbol{n}=\lambda \operatorname{tr}\left(\varepsilon_{0}^{t o t}\right) \boldsymbol{n}+2 \mu \boldsymbol{n} . \varepsilon_{0}^{t o t} \cdot \boldsymbol{n} .
\end{gathered}
$$


Substitution of (38), (39) into (33) yields after some algebra

$$
4 \mu\left(\mathcal{E}^{t o t}\right)^{2}+\frac{4 \mu^{2}}{\lambda+2 \mu}\left(e^{t o t}+\frac{\lambda}{2 \mu} \operatorname{tr}\left(\varepsilon_{0}^{t o t}\right)\right)^{2}=\frac{1-D_{0}}{\frac{1}{2}\left((2-\alpha) \kappa_{0}^{2}+\alpha \kappa_{0}\right) \bar{\psi}(\xi) F\left(\bar{y}_{0}\right)}
$$

where we have set

$$
\begin{gathered}
e^{t o t}=\boldsymbol{n} \cdot \varepsilon_{0}^{t o t} \cdot \boldsymbol{n} \\
\left(\mathcal{E}^{t o t}\right)^{2}=\left(\varepsilon_{0}^{t o t} \cdot \boldsymbol{n}\right) \cdot\left(\varepsilon_{0}^{t o t} \cdot \boldsymbol{n}\right)-\left(\boldsymbol{n} \cdot \varepsilon_{0}^{t o t} \cdot \boldsymbol{n}\right)^{2}
\end{gathered}
$$

$\mathcal{E}$ and e are respectively the tangent and normal components of the total strain vector in the direction $\boldsymbol{n}$. Therefore, the initial state of deformation $\varepsilon_{0}^{\text {tot }}$ maps the $(\mathrm{e}, \mathcal{E})$ plane into the Mohr circles of deformation for all the directions $\boldsymbol{n}$ and the bifurcation criterion maps into a set of ellipses in this plane. The size of the ellipses increases as the wave number $\xi$ increases. Before localization the smallest possible ellipse contains the largest Mohr circle. Bifurcation will occur for the first time when the smallest possible ellipse is tangent to the largest Mohr circle of deformation (the smallest possible ellipse corresponds to $\bar{\psi}(\xi)=1$ or equivalently to $\xi=0$ ). The normal vector $\boldsymbol{n}$ can be calculated geometrically from standard Mohr analysis (see Figure (B.2). After the first occurrence of bifurcation the ellipse will intersect the largest Mohr circle. In this case there is a finite set of normal vectors $\boldsymbol{n}$ such that bifurcation is possible. However, each vector $\boldsymbol{n}$ corresponds to the same ellipse whose radius is defined by $\xi$ (Figure (B.3)). The wavelength corresponding to each vector 
$\boldsymbol{n}$ is unique and it is obtained after (40)

$$
\begin{aligned}
& I(\boldsymbol{n})=\frac{2 \pi}{\xi}=\pi l_{c} \sqrt{2} \\
& / \sqrt{\log \left(\left(4 \mu\left(\mathcal{E}^{t o t}\right)^{2}+\frac{4 \mu^{2}}{\lambda+2 \mu}\left(\mathrm{e}^{t o t}+\frac{\lambda}{2 \mu} \operatorname{tr}\left(\varepsilon_{0}^{t o t}\right)\right)^{2}\right) \frac{\frac{1}{2}\left((2-\alpha) \kappa_{0}^{2}+\alpha \kappa_{0}\right) F\left(\bar{y}_{0}\right)}{\left(1-D_{0}\right)}\right)} .
\end{aligned}
$$

This wavelength is related to the width of the localization zone.

[Fig. 2 about here.]

The above relation can be written also in terms of the elasto-damage strain

$$
\begin{aligned}
& l(\boldsymbol{n})=\frac{2 \pi}{\xi}=\pi l_{c} \sqrt{2} \\
& / \sqrt{\log \left(\left(4 \mu\left(\mathcal{E}^{e l, d}\right)^{2}+\frac{4 \mu^{2}}{\lambda+2 \mu}\left(e^{e l, d}+\frac{\lambda}{2 \mu} \operatorname{tr}\left(\varepsilon_{0}^{e l, d}\right)\right)^{2}\right) \frac{\frac{1}{2}\left(2-\alpha+\frac{\alpha}{\kappa_{0}}\right) F\left(\bar{y}_{0}\right)}{\left(1-D_{0}\right)}\right)} .
\end{aligned}
$$

[Fig. 3 about here.]

Creep effect on the width of the localization zone

In order to study the effect of creep on the width of the localization zone, we consider two different load histories. The state variables will be denoted with the index $s c$ for the first one, and with index $m c$ for the second one, in which the ratio $\kappa_{0}$ of elasto-damage to total strain is smaller $\left(\kappa_{0}^{s c}>\kappa_{0}^{m c}\right)$.

We assume that at the bifurcation points of the two different histories, the 
elasto-damage strain is the same $\varepsilon_{0}^{(e l, d), m c}=\varepsilon_{0}^{(e l, d), s c}$, an assumption that yields $D_{0}^{m c}>D_{0}^{s c}$. Moreover, since the initial elasto-damage strain is the same for both loading paths one may prove that the normal $\boldsymbol{n}$ will be the same as well (Appendix B). Considering $\alpha$ constant and from (43), the wavelengths of the localized mode are such that $\left(1^{m c}(\boldsymbol{n})<1^{s c}(\boldsymbol{n})\right)$ as long as

$$
\frac{F\left(\bar{y}_{0}^{m c}\right)}{1-D_{0}^{m c}}>\frac{F\left(\bar{y}_{0}^{s c}\right)}{1-D_{0}^{s c}}
$$

or equivalently, in account of (9), as long as

$$
\frac{d \log F}{d \bar{y}} \leq-1
$$

Hence, provided the above conditions are fulfilled, the larger the creep strain at constant total strain, the smaller the wavelength of the localized mode. This decrease of wavelength provides also a decrease of the fracture process zone, an increase of brittleness and a shift on the size effect plot for geometrically similar specimens that is consistent with the experimental results in Fig.(B.1).

Role of $\alpha$ on the width of the localization zone

The role of $\alpha$, defined in Eqn. (18) is quite straightforward. If we replace $\alpha$ in (43) by $\alpha^{\prime}$ such that $\alpha^{\prime} \leq \alpha$, then we conclude that the width of the localization zone increases. In other words, if the contribution of the creep strain in the growth of damage decreases, the wavelength of the localized mode increases. This produces an increase of the size of the fracture process 
zone and an increase of ductility. For geometrically similar specimens, this increase of ductility is materialized by a shift of the experimental data point towards a strength criterion, to the left.

When damage depends solely upon the elastic energy $(\alpha=0)$, the bifurcation criterion becomes a function of only the elastic strain and creep effects can no longer be reproduced.

\section{Numerical example}

Consider the uniaxial initial state of strain

$$
\varepsilon_{0}^{t o t}=\left[\begin{array}{ccc}
\epsilon_{01}^{t o t} & 0 & 0 \\
0 & -\nu \epsilon_{01}^{t o t} & 0 \\
0 & 0 & -\nu \epsilon_{01}^{t o t}
\end{array}\right]
$$

The critical wavelengths can be obtained when the ellipse corresponding to the above initial state of deformation is tangent to the largest Mohr circle leading to the localization criterion

$$
\begin{aligned}
& \frac{E(1+\nu)}{2}\left(\epsilon_{01}^{t o t} \sin (2 \theta)\right)^{2} \\
& +\frac{E(1-2 \nu)(1+\nu)}{4(1-\nu)}(1+\cos (2 \theta))^{2}\left(\epsilon_{01}^{t o t}\right)^{2} \\
& \quad-\frac{\left(1-D_{0}\right)}{\frac{1}{2}\left((2-\alpha) \kappa_{0}^{2}+\alpha \kappa_{0}\right) F\left(\bar{y}_{0}\right) \bar{\psi}(\xi)}=0
\end{aligned}
$$


where the angle $\theta$ defines the normal $\boldsymbol{n}=(\cos \theta, 0, \sin \theta)$ to the localization band and satisfies

$$
\tan ^{2}(2 \theta)=\frac{2 \frac{E(1-\nu)(1+\nu)}{1-2 \nu}\left((2-\alpha) \kappa_{0}^{2}+\alpha \kappa_{0}\right) F\left(\bar{y}_{0}\right) \bar{\psi}(\xi)\left(\epsilon_{01}^{t o t}\right)^{2}-\left(1-D_{0}\right)}{\left(1-D_{0}\right)-E(1+\nu)\left((2-\alpha) \kappa_{0}^{2}+\alpha \kappa_{0}\right) F\left(\bar{y}_{0}\right) \bar{\psi}(\xi)\left(\epsilon_{01}^{t o t}\right)^{2}}
$$

as it can be proved with Mohr analysis.

[Fig. 4 about here.]

We particularize function $F$, for illustration purposes, as

$$
F(\bar{y})=\frac{b_{1}+2 b_{2}\left(\bar{y}-y^{0}\right)}{\left(1+b_{1}\left(\bar{y}-y^{0}\right)+b_{2}\left(\bar{y}-y^{0}\right)^{2}\right)^{2}},
$$

where the numerical values of the model parameters are $E=32.000 \mathrm{MPa}$, $\nu=0.2, b_{1}=605 \mathrm{MPa}^{-1}, b_{2}=5.4210^{4} \mathrm{MPa}^{-2}$ and $y^{0}=610^{-5} \mathrm{MPa}$. Damage is then given by

$$
D(\bar{y})=1-\frac{1}{1+b_{1}\left(\bar{y}-y^{0}\right)+b_{2}\left(\bar{y}-y^{0}\right)^{2}}
$$

As we have an infinite body $\bar{y}=y=\frac{1}{2}\left(1-\alpha+\alpha / \kappa_{0}\right) E\left(\epsilon_{01}^{t o t}\right)^{2}$. Function $F$ satisfies criterion (45) for $y \leq y_{c r}=2.995$. $y_{c r}$ yields the damage value $D_{c r} \simeq 0.999998$ (Figure (B.4)).

[Fig. 5 about here.]

Solving (47) for $\bar{\psi}(\xi)$ and substituting in (46) yields

$$
\begin{aligned}
\frac{E(1-2 \nu)(1+\nu)}{4(1-\nu)}(1+\cos (2 \theta))^{2}-4 \frac{E(1-\nu)(1+\nu)}{1-2 \nu} \cos ^{2}(2 \theta) & \\
& +\frac{5 E(1+\nu)}{2} \sin ^{2}(2 \theta)=0 .
\end{aligned}
$$


Thus, the direction of the normal $\boldsymbol{n}$ to the localization band does not depend, in the uniaxial case, upon the state of strain or on the damage or on the yield function of damage but only upon the elastic parameters of the material. Solving (50), one gets $\theta=\pi / 4$. This angle corresponds to a direction of localization in the $\left(x_{1}, x_{2}\right)$ or $\left(x_{1}, x_{3}\right)$ planes.

[Fig. 6 about here.]

[Fig. 7 about here.]

Figure (B.5) shows the admissible wavelengths normalized to the characteristic length $l_{c}$ as a function of the initial state of damage $D_{0}$ for a uniaxial time-independent process ( $\alpha=0$ and $\kappa_{0}=1$ ). In Figure (B.6) the normalized admissible wavelengths of the proposed model at the initial uniaxial elastodamage state of strain $\epsilon_{03}^{e l, d}=\epsilon_{02}^{e l, d}=-\nu \epsilon_{01}^{e l, d}=-0.00032 \nu$ are plotted against the initial state of damage $D_{0}$ for $\alpha=0.2$. We see that as $D_{0}$ increases, i.e. as the creep strains increase, the admissible wavelengths decrease. Moreover, when the parameter $\alpha$ decreases the normalized admissible wavelengths increase (Figure (B.7)). In Figure (B.7) the admissible wavelengths are plotted against the initial state of total strain $\epsilon_{03}^{\text {tot }}=\epsilon_{02}^{t o t}=-\nu \epsilon_{01}^{\text {tot }}=-0.00032 \nu$ because against the initial damage $D_{0}$ the difference is negligible. 


\section{Conclusion}

The localization properties of a rate-dependent material described by a nonlocal damage-based constitutive relation coupled to linear viscoelasticity has been investigated. The localization condition is similar to that of the rateindependent underlying damage model. The normal $\boldsymbol{n}$ to the localization band at the onset of bifurcation depends only on the state of elastic strain and not on the creep strain.

The proposed coupled model preserves localization limiting properties, same as in the rate independent case (Pijaudier-Cabot and Benallal, 1993) and it is consistent with the effect of creep observed experimentally. Namely, the minimum wavelength of the localization modes cannot be zero in accordance with energy considerations and the admissible wavelengths decrease when the creep effect (strains) increases, at least under a certain condition on the growth of the yield function of damage. This last property should induce a variation on size effect data for geometrically similar specimens: a shift in the size effect plot towards increasing brittleness for an increasing creep strain. Moreover, when the fraction of creep strain which contributes to damage is decreasing, a shift on the size effect plot occurs towards the left hand side, i.e. towards the strength criterion and an increase of ductility is expected. The model fails to predict the influence of creep on the wavelength of localized modes when damage is considered to depend solely upon the elastic energy. 
Such analytical results are expected to be recovered in computational failure analyses. They are expected to have important practical consequences: as creep develops, the residual capacity of a given structure may decrease; the structure is becoming more brittle, safety margins may be reduced. This of course should hold for severely loaded concrete structures, where damage interacts with creep. It may typically occur in some regions of prestressed concrete structures subjected to stress concentrations.

\section{A}

Consider a time-independent process until the column matrix stress takes the value $\boldsymbol{\sigma}_{\text {ind }}$ and then the loading state remains fixed for the time interval $\left[t_{\text {ind }}, t_{0}\right]$, where $t_{0}$ is the time at which bifurcation occurs. For this loading path relations (4) would give

$$
\varepsilon_{0}^{c r}=\sum_{i=1}^{N} \varepsilon_{i_{0}}^{c r}=\sum_{i=1}^{N} \frac{1-e^{-t / \tau_{i}}}{E_{i}} \mathbf{C}_{\nu}^{-1} \boldsymbol{\sigma}_{i n d} .
$$

Since

$$
\varepsilon_{0}^{e l, d}=\frac{1}{\left(1-D_{0}\right) E} \mathbf{C}_{\nu}^{-1} \boldsymbol{\sigma}_{i n d}
$$

one may conclude that (32) holds true for

$$
\kappa_{0}=\frac{1 /\left(\left(1-D_{0}\right) E\right)}{1 /\left(\left(1-D_{0}\right) E\right)+\sum_{i=1}^{N}\left(1-e^{-t / \tau_{i}}\right) / E_{i}} .
$$

In the same spirit, relation (32) can be proved for more general loading paths for which the loading state is either fixed or changes through a time- 
independent process.

In the uniaxial loading now, at the onset of bifurcation, relation (6) reads

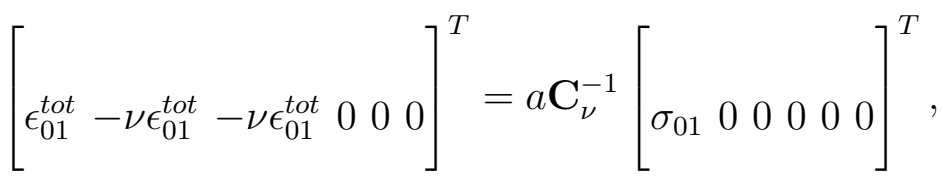

that is

$$
\varepsilon_{0}^{t o t}=\mathbf{C}_{\nu}^{-1} a \boldsymbol{\sigma}_{0}
$$

where for simplicity we assumed $t^{\prime}=0$ and $a$ is a number such that

$$
a \boldsymbol{\sigma}_{0}=\int_{0}^{t_{0}} J(t, \tau) d \boldsymbol{\sigma}(\tau)
$$

Moreover,

$$
\varepsilon_{0}^{e l, d}=\frac{1}{\left(1-D_{0}\right) E} \mathbf{C}_{\nu}^{-1} \boldsymbol{\sigma}_{0}
$$

holds true. Using now (A.5) and (A.6), we deduce (32) with $\kappa_{0}=1 /((1-$ $\left.\left.D_{0}\right) E a\right)$

B

The normal $\boldsymbol{n}=(\cos \theta, 0, \sin \theta)$ to the localization band can be obtained when the ellipse corresponding to the initial state of strain $\varepsilon_{0}^{\text {tot }}$ is tangent to the largest Mohr circle leading to system

$$
\tan ^{2}(2 \theta)=\frac{2(\lambda+2 \mu)\left((2-\alpha) \kappa_{0}^{2}+\alpha \kappa_{0}\right) F\left(\bar{y}_{0}\right) \bar{\psi}(\xi)\left(\epsilon_{01}^{t o t}-\epsilon_{03}^{t o t}\right)^{2}-\left(1-D_{0}\right)}{\left(1-D_{0}\right)-2 \mu\left((2-\alpha) \kappa_{0}^{2}+\alpha \kappa_{0}\right) F\left(\bar{y}_{0}\right) \bar{\psi}(\xi)\left(\epsilon_{01}^{t o t}-\epsilon_{03}^{t o t}\right)^{2}}
$$




$$
\begin{gathered}
4 \mu\left(\frac{\epsilon_{01}^{t o t}-\epsilon_{03}^{t o t}}{2} \sin (2 \theta)\right)^{2}+\frac{4 \mu^{2}}{\lambda+2 \mu}\left(\frac{\epsilon_{01}^{t o t}+\epsilon_{03}^{t o t}}{2}+\frac{\epsilon_{01}^{t o t}-\epsilon_{03}^{t o t}}{2} \cos (2 \theta)+\frac{\lambda}{2 \mu} \operatorname{tr}\left(\varepsilon_{0}^{t o t}\right)\right)^{2} \\
=\frac{1-D_{0}}{\frac{1}{2}\left((2-\alpha) \kappa_{0}^{2}+\alpha \kappa_{0}\right) \bar{\psi}(\xi) F\left(\bar{y}_{0}\right)}
\end{gathered}
$$

Solving (B.1) for $\bar{\psi}(\xi)$ and substituting into (B.2) yields

$$
\begin{array}{r}
4 \mu\left(\frac{\epsilon_{01}^{t o t}-\epsilon_{03}^{t o t}}{2} \sin (2 \theta)\right)^{2}+\frac{4 \mu^{2}}{\lambda+2 \mu} \\
\left(\frac{\epsilon_{01}^{t o t}+\epsilon_{03}^{t o t}}{2}+\frac{\epsilon_{01}^{t o t}-\epsilon_{03}^{t o t}}{2} \cos (2 \theta)+\frac{\lambda}{2 \mu} \operatorname{tr}\left(\varepsilon_{0}^{t o t}\right)\right)^{2} \\
=4 \frac{\lambda+2 \mu+\mu \tan ^{2}(2 \theta)}{1+\tan ^{2}(2 \theta)}\left(\epsilon_{01}^{t o t}-\epsilon_{03}^{t o t}\right)^{2}
\end{array}
$$

If (32) is satisfied, the above equation (B.3) reads

$$
\begin{aligned}
4 \mu\left(\frac{\epsilon_{01}^{e l, d}-\epsilon_{03}^{e l, d}}{2} \sin (2 \theta)\right)^{2} & \\
+\frac{4 \mu^{2}}{\lambda+2 \mu}\left(\frac{\epsilon_{01}^{e l, d}+\epsilon_{03}^{e l, d}}{2}+\right. & \left.\frac{\epsilon_{01}^{e l, d}-\epsilon_{03}^{e l, d}}{2} \cos (2 \theta)+\frac{\lambda}{2 \mu} \operatorname{tr}\left(\varepsilon_{0}^{e l, d}\right)\right)^{2} \\
& =4 \frac{\lambda+2 \mu+\mu \tan ^{2}(2 \theta)}{1+\tan ^{2}(2 \theta)}\left(\epsilon_{01}^{e l, d}-\epsilon_{03}^{e l, d}\right)^{2} .
\end{aligned}
$$

It is then obvious from (B.4) that, as long as (32) holds true, the normal $\boldsymbol{n}$ remains fixed for fixed initial elasto-damage strain $\varepsilon_{0}^{e l, d}$.

\section{References}

Z. P. Bažant. Stable states and paths of structures with plasticity or damage. Journal of Engineering Mechanics, 114(12):2013-2034, 1988.

Z. P. Bažant. Current status and advances in the theory of creep and interaction with fracture. In Z.P. Bažant and I. Carol, editors, Proc. 5th Int. RILEM Symp. on Creep and Shrinkage of Concrete, pages 291-307, Barcelona, 1993. E\&FN Spon, London. 
Z. P. Bažant and R. Gettu. Rate effects and load relaxation: static fracture of concrete. ACI Materials Journal, 89(5):456-468, 1992.

Z. P. Bažant and Y. N. Li. Cohesive crack with rate-dependent opening and viscoelasticity: I. mathematical model and scaling. International Journal of Fracture, 86:247-265, 1997.

Z. P. Bažant and G. Pijaudier-Cabot. Nonlocal continuum damage, localization instability and convergence. Journal of Applied Mechanics ASME, 55: 287-294, 1988.

Z. P. Bažant and J. Planas. Fracture and size Effect in Concrete and Other Quasi-Brittle Materials. Boca Raton and London, 1998.

A. Benallal. Localization phenomena in thermo-elastoplasticity. Arch. Mech., 44(1):15-29, 1992.

R. de Borst, A.H. Van Den Boogaard, and L.J. Sluys. Computational issues in time-dependent deformation and fracture of concrete. In Z.P. Bažant and I. Carol, editors, Proc. 5th Int. RILEM Symp. on Creep and Shrinkage of Concrete, pages 309-325, Barcelona, 1993. E\&FN Spon, London.

J.-F. Dubé, G. Pijaudier-Cabot, and C. La Borderie. Rate dependent damage model for concrete in dynamics. Journal of Engineering Mechanics, 122: 939-947, 1996.

J. Hadamart. Leçons sur la Propagation des Ondes. France, 1903.

K. Haidar, G. Pijaudier-Cabot, J.-F. Dubé, and A. Loukili. Correlation between internal length, fracture process zone and size effect in mortar and model materials. Materials and Structures, 38:201-210, 2005. 
R. Hill. Some basic principles in the mechanics of solids without natural time. J. Mech. Phys. Solids, 7:209-225, 1959.

J. Lemaitre and J.L. Chaboche. Mécanique des matériaux solides. Dunod, Paris, 1985.

B. Loret and J.H. Prevost. Dynamic strain localization in fluid saturated porous media. In Proc. 2nd World Congress on Computational Mechanics, pages 278-281, 1990.

C. Mazzotti and M. Savoia. Experimental study of nonlinear creep of concrete at high stress levels. In Z.P. Bažant, F.-J. Ulm, and F.H. Whitmann, editors, Proc. Concreep 6, pages 259-264, Cambridge, England, 2001. Elsevier, Amsterdam.

C. Mazzotti and M. Savoia. Nonlinear creep damage model for concrete under uniaxial compression. Journal of Engineering Mechanics, 129:1065-1075, 2003.

A. Needleman. Material rate dependence and mesh sensitivity in localization problems. Comput. Meth. Appl. Mech. Engng, 87:64-85, 1988.

M. Omar, G. Pijaudier-Cabot, and A. Loukili. Etude comparative du couplage endommagement - fluage. Revue Française de Génie Civil, 8:457-482, 2004.

G. Pijaudier-Cabot and Z. Bažant. Nonlocal damage theory. Journal of Engineering Mechanics, 113:1512-1533, 1987.

G. Pijaudier-Cabot and A. Benallal. Strain localization and bifurcation in a nonlocal continuum. International Journal of Solids and Structures, 30: 1761-1775, 1993. 
J. R. Rice. The localization of plastic deformation, theoritical and applied mechanics. In W.T. KOTER, editor, Proc. 14th IUTAM Congr., Delft, the Netherlands, 30 August-4 September 1976. North Holland, Amsterdam.

J. C. Rots. Computational Modelling of Concrete Fracture. PhD thesis, Delft University of Technology, Delft, the Netherlands, 1988.

J. W. Rudnicki and J. R. Rice. Conditions for the localization of deformation in pressure sensitive dilatant materials. J. Mech. Phys. Solids, 23:371-394, 1975.

H. Rüsch. Research toward a general flexural theory for structural concrete. ACI journal, 57(1):1-28, 1960.

J. C. Simo and D. D. Fox. On a stress resultant geometrically exact shell model, part i, formulation and optimal parametrization. Computer Methods in Applied Mechanics and Engineering, 72:267-304, 1989. 


\section{List of Figures}

B.1 Nominal strengths of 4 groups of 3 specimens of different sizes tested at 4 different times to peak (after Bažant and Gettu 1992)

B.2 Geometrical solution to the bifurcation problem: Critical state.

B.3 Geometrical solution to the bifurcation problem: Solutions after the first bifurcation.

B.4 Evolution of $\frac{d \log F}{d \bar{y}}$ in a time-independent process $(\alpha=0$ and $\kappa_{0}=1$ ). Criterion (45) is satisfied for $y \leq y_{c r}=2.995$.

B.5 Uniaxial tension: normalized admissible wavelengths of the localization mode as a function of the initial state of damage for a time-independent process $\left(\alpha=0\right.$ and $\left.\kappa_{0}=1\right)$.

B.6 Uniaxial tension: normalized admissible wavelengths of the localization mode as a function of the initial state of damage $D_{0}$ for fixed initial state of elasto-damage strain $\epsilon_{03}^{e l, d}=\epsilon_{02}^{e l, d}=-\nu \epsilon_{01}^{e l, d}=-0.00032 \nu$ and for $\alpha=0.2$. Shift on the size-effect plot towards LEFM with increasing $D_{0}$.

B.7 Uniaxial tension: normalized admissible wavelengths of the localization mode for two different values of $\alpha$ for fixed initial state of elasto-damage strain $\epsilon_{03}^{e l, d}=\epsilon_{02}^{e l, d}=-\nu \epsilon_{01}^{e l, d}=$ $-0.00032 \nu$. Shift on the size-effect plot towards the strength criterion with decreasing $\alpha$. 


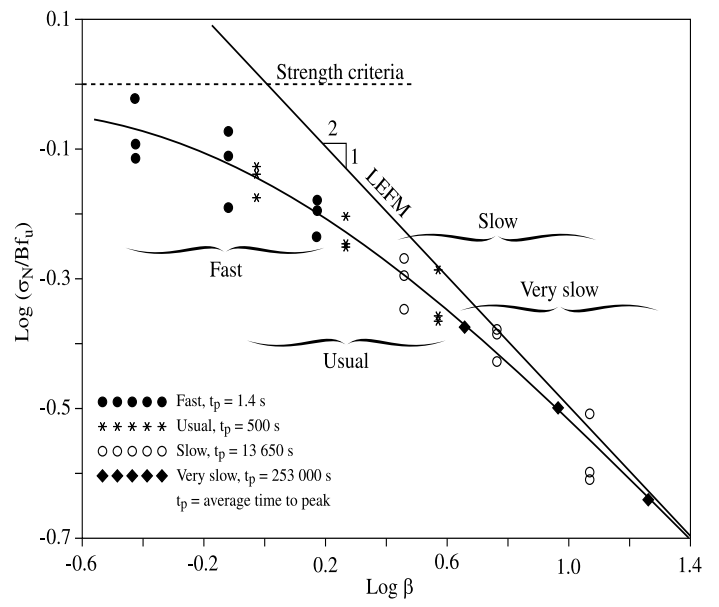

Fig. B.1. Nominal strengths of 4 groups of 3 specimens of different sizes tested at 4 different times to peak (after Bažant and Gettu 1992) 


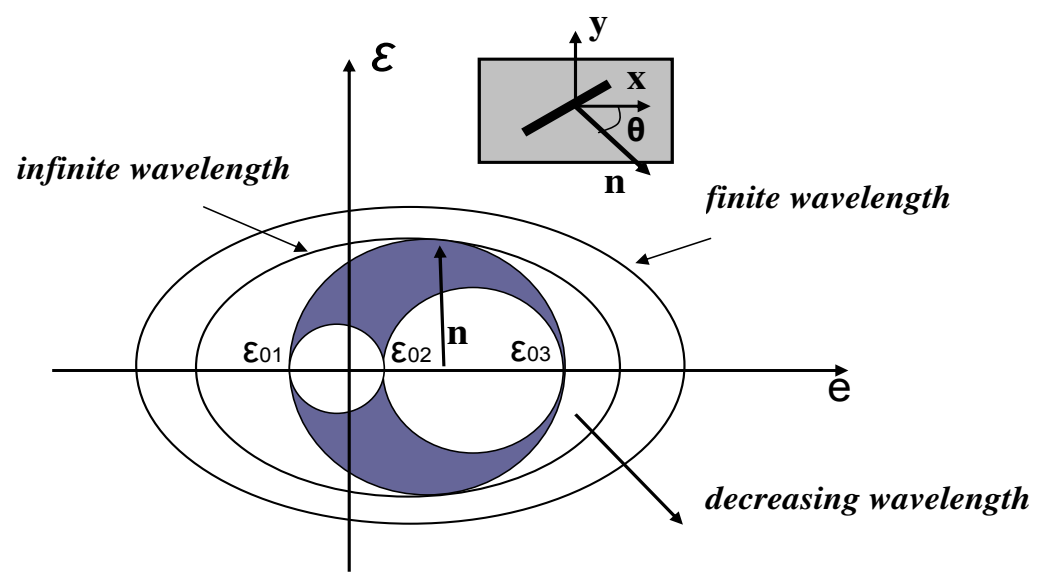

Fig. B.2. Geometrical solution to the bifurcation problem: Critical state. 




Fig. B.3. Geometrical solution to the bifurcation problem: Solutions after the first bifurcation. 


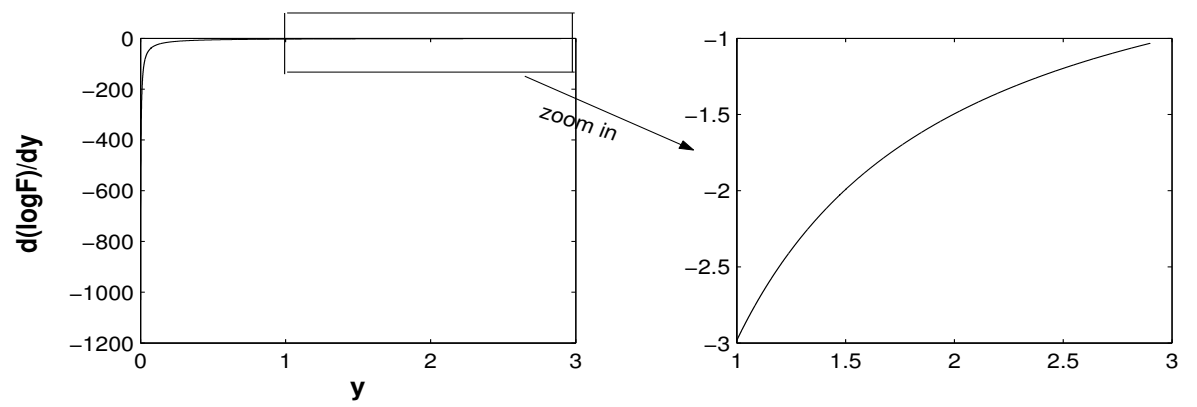

Fig. B.4. Evolution of $\frac{d \log F}{d \bar{y}}$ in a time-independent process $\left(\alpha=0\right.$ and $\left.\kappa_{0}=1\right)$. Criterion (45) is satisfied for $y \leq y_{c r}=2.995$. 


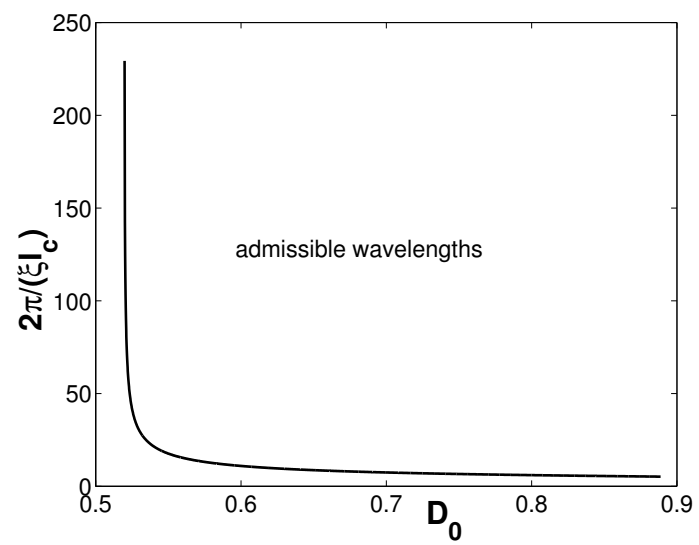

Fig. B.5. Uniaxial tension: normalized admissible wavelengths of the localization mode as a function of the initial state of damage for a time-independent process $\left(\alpha=0\right.$ and $\left.\kappa_{0}=1\right)$. 


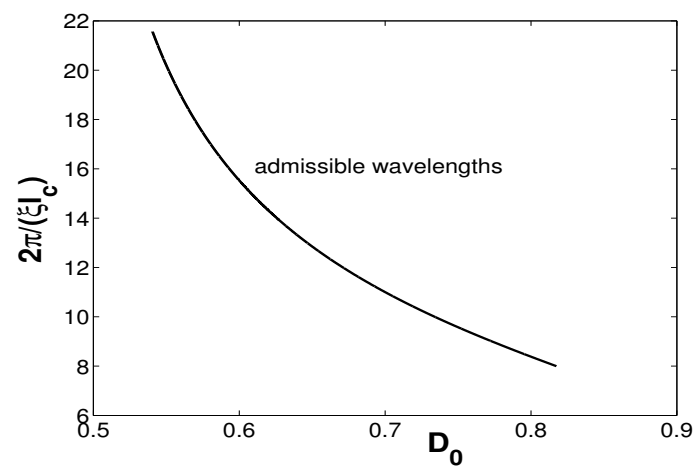

Fig. B.6. Uniaxial tension: normalized admissible wavelengths of the localization mode as a function of the initial state of damage $D_{0}$ for fixed initial state of elasto-damage strain $\epsilon_{03}^{e l, d}=\epsilon_{02}^{e l, d}=-\nu \epsilon_{01}^{e l, d}=-0.00032 \nu$ and for $\alpha=0.2$. Shift on the size-effect plot towards LEFM with increasing $D_{0}$. 


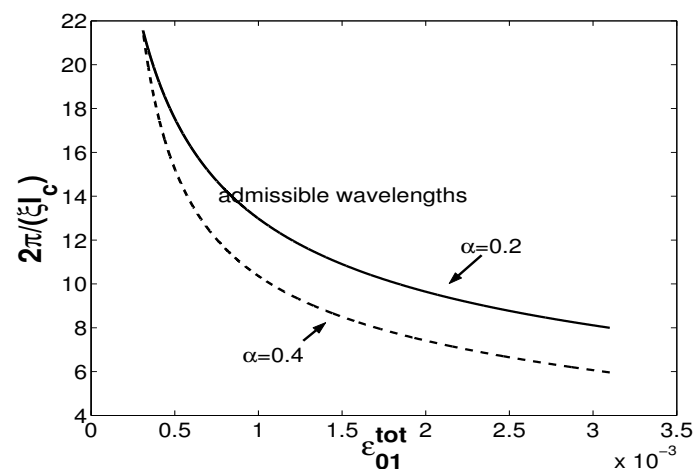

Fig. B.7. Uniaxial tension: normalized admissible wavelengths of the localization mode for two different values of $\alpha$ for fixed initial state of elasto-damage strain $\epsilon_{03}^{e l, d}=\epsilon_{02}^{e l, d}=-\nu \epsilon_{01}^{e l, d}=-0.00032 \nu$. Shift on the size-effect plot towards the strength criterion with decreasing $\alpha$. 